\title{
Okuläre Nebenwirkungen von biologischen Anti-TNF-Medikamenten - eine Übersicht
}

\author{
Fernanda Nicolela Susanna $^{a}$ Carlos Pavesio $^{b}$ \\ ${ }^{a}$ Medicine School, University of São Paulo, São Paulo, Brasilien; \\ ${ }^{\text {b}}$ Moorfields Eye Hospital NHS Foundation Trust and UCL Institute of Ophthalmology, London, UK
}

\author{
Schlüsselwörter \\ Biologika · Anti-TNF · Uveitis · Optikusneuritis · okuläre \\ Nebenwirkung · entzündliche Augenerkrankung
}

\section{Zusammenfassung}

Die kürzlich erfolgte Einführung biologischer Wirkstoffe hat die Behandlung chronischer immuninflammatorischer Erkrankungen revolutioniert, doch ist diese neue Therapie mit erheblichen Nebenwirkungen verbunden.

Durch groß angelegte kontrollierte Studien, in denen sich ein Rückgang der Uveitis-Schübe zeigte, hat die Therapie mit Tumornekrosefaktor (TNF)-Hemmern bei nicht infektiöser Uveitis weiter an Bedeutung gewonnen. Es liegen Berichte vor, denen zufolge diese Medikamente paradoxerweise entgegen ihrer therapeutischen Wirkung mit dem Auftreten oder Wiederauftreten von entzündlichen Augenerkrankungen assoziiert sind.

Etliche Studien deuten darauf hin, dass Anti-TNF-a-Wirkstoffe bei der Auslösung oder Verschlimmerung eines zugrunde liegenden Entzündungsprozesses eine Rolle spielen könnten, darunter auch die Hypothese eines gestörten Zytokingleichgewichts; die Mechanismen, die für diese Nebenwirkungen verantwortlich sind, sind jedoch noch nicht vollständig geklärt.

Es erfolgte eine Literatursuche in PubMed anhand folgender Begriffe: ophthalmologische Komplikationen, Uveitis, entzündliche Augenerkrankung, Optikusneuritis, Neuropathie, Nebenwirkun- gen, Anti-TNF, TNF-alpha-Inhibitor, Infliximab, Etanercept, Adalimumab, Golimumab, Certolizumab und Biologika. Die in der vorliegenden Studie vorgestellten Daten stammen überwiegend aus der Anwendung von TNF-Inhibitoren in der Rheumatologie, was vor allem daran liegt, dass diese Medikamente in diesem Fachgebiet schon seit längerer Zeit eingesetzt werden.

Viele der in dieser Übersichtsarbeit berichteten okulären Nebenwirkungen können möglicherweise als paradoxe Wirkung der AntiTNF-Therapie betrachtet werden. Wir fanden eine Vielzahl von Daten, nach denen eine Anti-TNF-Therapie bei rheumatischen Erkrankungen mit einer neu auftretenden Uveitis assoziiert war, insbesondere unter Etanercept.

Insgesamt lässt sich sagen, dass zwar zunehmend Daten zu okulären Nebenwirkungen vorliegen, doch bleibt abzuwarten, ob der vermutete Zusammenhang zwischen TNF-Inhibitoren und dem Auftreten von Augenentzündungen durch weitere qualitativ hochwertige Daten untermauert wird. Gleichwohl soll das Bewusstsein für mögliche Nebenwirkungen der Anti-TNF-Therapie erhöht werden.

(C) 2020 The Author(s)

\section{Hintergrund}

Entzündliche Autoimmunerkrankungen betreffen etwa 7,6 bis 9,4\% der Weltbevölkerung, insbesondere junge Frauen und Frauen mittleren Alters. Diese Krankheiten gehören weltweit zu den häufigsten Todesursachen und sind häufig mit schwerer und chronischer Morbidität verbunden [1].
Die Einführung biologischer Wirkstoffe hat die Behandlung der chronischen immunvermittelten entzündlichen Erkrankungen wie etwa der chronisch entzündlichen rheumatischen Erkrankungen und unlängst auch der Uveitis in den vergangenen zwei Jahrzehnten revolutioniert [2]. Zwar bestehen Unterschiede im Hinblick auf das klinische Bild und die Pathophysiologie, doch spielt bei allen diesen Krankheiten der Tumornekrosefaktor 
(TNF) als wichtiges proinflammatorisches Zytokin eine Rolle bei der Pathogenese, und sie sprechen tendenziell positiv auf Medikamente an, die ihn gezielt angreifen [3].

Anti-TNF-Medikamente sind meist gut verträglich. Mit steigender Zahl biologischer Therapien ist jedoch eine Vielzahl von Nebenwirkungen aufgetreten, deren Mechanismen noch nicht vollständig geklärt sind. Die wichtigste Nebenwirkung sind Reaktionen an der Infusions- oder Injektionsstelle, doch sind diese in der Regel beherrschbar. Zu den selteneren, jedoch potenziell schwerwiegenderen Komplikationen gehören die Reaktivierung einer Tuberkulose und eine erhöhte Inzidenz anderer Infektionen sowie Zytopenien oder, selten, eine aplastische Anämie, ein erhöhtes Malignomrisiko, die Verschlimmerung einer kongestiven Herzinsuffizienz, Hepatotoxizität und Exazerbation oder Auftreten einer demyelinisierenden Erkrankung [4]. Darüber hinaus wurden paradoxe inflammatorische Effekte von TNF-aInhibitoren beobachtet, darunter die Exazerbation oder Entwicklung von medikamenteninduzierten Autoimmunerkrankungen wie Psoriasis, systemischem Lupus erythematodes, Vaskulitis und Uveitis [5].

In der vorliegenden Übersichtsarbeit liegt der Schwerpunkt auf einem weniger gut dokumentierten Aspekt dieser Therapie: den okulären Nebenwirkungen. Diese reichen von einer gewöhnlichen Bindehautentzündung bis zur Endophthalmitis und können potenziell zum Verlust des Sehvermögens führen. Dies zeigt, wie wichtig es ist, okuläre Manifestationen bei Patienten, die AntiTNF- $\alpha$-Wirkstoffe erhalten, engmaschig zu überwachen.

Es sei darauf hingewiesen, dass bei der Beurteilung des Sicherheitsprofils die gewonnenen Ergebnisse je nach Grunderkrankung unterschiedlich ausfallen, und diese kann für die Häufigkeit sowie für die Art und Schwere der Nebenwirkungen von entscheidender Bedeutung sein [6].

\section{Ziel}

Mit der vorliegenden Studie sollte die derzeitige Evidenzlage zu okulären Nebenwirkungen von Anti-TNF-Wirkstoffen untersucht werden.

\section{Methoden}

Es erfolgte eine Literatursuche in PubMed. Die Suche war auf die neueste Literatur ausgerichtet, schloss jedoch alle nach 1990 veröffentlichten Artikel ein. Die für die Suche verwendeten Schlüsselwörter waren: ophthalmologische Komplikation, Uveitis, entzündliche Augenerkrankung, Optikusneuritis, Neuropathie, Nebenwirkungen, Anti-TNF, TNF-alpha-Inhibitor, Infliximab, Etanercept, Adalimumab, Golimumab, Certolizumab und Biologika. In diese Übersichtsarbeit wurden überwiegend Studien aus anderen medizinischen Fachgebieten als Augenheilkunde eingeschlossen. Da TNF-Inhibitoren zuerst in anderen Fachgebieten wie etwa der Rheumatologie zum Einsatz kamen, werden bereits seit langer Zeit große Patientenserien behandelt, und die Evidenzqualität der Literatur ist in diesen Fachgebieten höher. Die Litera- turangaben in den ausgewählten Manuskripten wurden nach weiteren relevanten Studien durchsucht.

\section{Anti-TNF-Wirkstoffe}

Bei TNF- $\alpha$ handelt es sich um ein homotrimerisches Transmembranprotein von $26 \mathrm{kDa}$, das auf der Oberfläche von Makrophagen, T-Lymphozyten, natürlichen Killerzellen, glatten Muskelzellen sowie Fibroblasten exprimiert wird [7]. Im gesunden Organismus spielt TNF eine wichtige Rolle bei der Initiierung der Entzündungsreaktion gegen eingedrungene Krankheitserreger [8]. Bei immunvermittelten Krankheiten führen dagegen hohe TNF-Konzentrationen über direkte pathogenetische Effekte, die Bildung weiterer Entzündungsmediatoren, Apoptose und Gewebezerstörung zu einer überschießenden systemischen Entzündung und Organschädigung [9]. Die Senkung der TNF-aKonzentration durch Anti-TNF-Wirkstoffe bewirkt einen Rückgang der chronischen pathologischen Entzündungsreaktionen [10], wodurch das Behandlungsergebnis entzündlicher Autoimmunerkrankungen deutlich verbessert wird [11].

Diese Art der Therapie umfasst Medikamente, die strukturell aus monoklonalen Antikörpern, ihren Fab-Anteilen oder sogar Antikörper-Fusionsproteinen bestehen, die gegen Zytokine und Membranrezeptoren gerichtet sein können $[12,13]$. Derzeit gibt es $2 \mathrm{Ar}-$ ten von Anti-TNF-Therapien: monoklonale Antikörper und lösliche Rezeptoren sowie 6 verschiedene Medikamente (Tab. 1) [14].

\section{Anti-TNF-Therapie in der Ophthalmologie}

Die Uveitis ist eine häufig auftretende Augenerkrankung, die das Sehvermögen gefährden kann und allgemein als Entzündung der Uvea beschrieben wird. Sie kann infektiöse und nicht-infektiöse Ursachen haben und ist für 5 bis 10\% der weltweiten Sehbehinderungen verantwortlich [15]. In den USA gilt sie als dritthäufigste Ursache von vermeidbarer Erblindung [16].

Grundlage der derzeitigen Standardbehandlung in Zentren, die nicht auf Uveitis spezialisiert sind, ist die Kortikosteroidgabe zur Bekämpfung der Entzündung [16]. Zwar sind sowohl topische als auch systemische Kortikosteroide akut wirksam, doch ist die Langzeitanwendung dieser Medikamente mit schwerwiegenden Nebenwirkungen verbunden, was die Suche nach Behandlungsalternativen vorangetrieben hat.

TNF-Inhibitoren erwiesen sich zunehmend als eine mögliche Therapie bei Uveitis, da etliche groß angelegte kontrollierte Studien darauf hindeuteten, dass bei Patienten mit immunvermittelten Erkrankungen, die Anti-TNF- $\alpha$-Wirkstoffe erhielten, die Zahl der Uveitisschübe signifikant zurückging [17]. Die Ergebnisse fielen unter Infliximab und Adalimumab deutlicher aus als unter Etanercept [17].

Die Studien VISUAL I und II zeigten, dass Adalimumab in der Lage ist, die Zahl der Uveitisschübe im Vergleich zu Placebo zu verringern (28\% und $20 \%$ für VISUAL I bzw. II) $[18,19]$ und eine Verringerung der Kortikosteroiddosis über einen Zeitraum von 80 Wochen ermöglichte. Diese Ergebnisse bedeuteten einen Wen- 
Tab. 1. Monoklonale Antikörper

\begin{tabular}{|c|c|c|c|c|c|}
\hline Medikament & Etanercept (Enbrel ${ }^{\circledR}$ ) & Infliximab (Remicade ${ }^{\circledR}$ ) & Adalimumab (Humira ${ }^{\circledR}$ ) & $\begin{array}{l}\text { Golimumab (Simponi }{ }^{\circledR} \text {, } \\
\left.\text { Simponi Aria }{ }^{\circledR}\right)\end{array}$ & $\begin{array}{l}\text { Certolizumab } \\
\left(\text { Cimzia }^{\circledR}\right)\end{array}$ \\
\hline $\begin{array}{l}\text { Struktur und } \\
\text { Wirkmechanismus }\end{array}$ & $\begin{array}{l}\text { TNFR2-Ektodomäne } \\
\text { fusioniert mit IgG1, } \\
\text { bindet TNF-a und TNF- } \beta\end{array}$ & $\begin{array}{l}\text { Chimäres murin/humanes } \\
\text { lgG1, das an TNF-a bindet }\end{array}$ & $\begin{array}{l}\text { Vollständig humanisiertes } \\
\text { lgG1, das an TNF-a bindet }\end{array}$ & $\begin{array}{l}\text { Vollständig } \\
\text { humanisiertes lgG1, } \\
\text { das an TNF-a bindet }\end{array}$ & $\begin{array}{l}\text { Humanisiertes } \\
\text { pegylierters Fab- } \\
\text { Fragment, das an } \\
\text { TNF-a bindet }\end{array}$ \\
\hline Indikationen & $\begin{array}{l}\text { Rheumatoide Arthritis, } \\
\text { Psoriasis-Arthritis, Plaque- } \\
\text { Psoriasis, ankylosierende } \\
\text { Spondylitis und juvenile } \\
\text { idiopathische Arthritis }\end{array}$ & $\begin{array}{l}\text { Rheumatoide Arthritis, } \\
\text { Psoriasis-Arthritis, Plaque- } \\
\text { Psoriasis, ankylosierende } \\
\text { Spondylitis, idiopathische } \\
\text { Lungenfibrose, Colitis } \\
\text { ulcerosa und Morbus } \\
\text { Crohn }\end{array}$ & $\begin{array}{l}\text { Rheumatoide Arthritis, } \\
\text { Psoriasis-Arthritis, } \\
\text { ankylosierende Spondylitis, } \\
\text { Spondyloarthritis, juvenile } \\
\text { idiopathische Arthritis, } \\
\text { Uveitis (intermediär, } \\
\text { posterior, Panuveitis), } \\
\text { Plaque-Psoriasis, Hidradenitis } \\
\text { suppurativa, Colitis ulcerosa } \\
\text { und Morbus Crohn }\end{array}$ & $\begin{array}{l}\text { Rheumatoide Arthritis, } \\
\text { Psoriasis-Arthritis, } \\
\text { ankylosierende } \\
\text { Spondylitis und Colitis } \\
\text { ulcerosa }\end{array}$ & $\begin{array}{l}\text { Rheumatoide } \\
\text { Arthritis, Psoriasis- } \\
\text { Arthritis, } \\
\text { ankylosierende } \\
\text { Spondylitis, } \\
\text { Spondyloarthritis } \\
\text { und Morbus Crohn }\end{array}$ \\
\hline $\begin{array}{l}\text { Berichtete okuläre } \\
\text { Nebenwirkungen }\end{array}$ & $\begin{array}{l}\text { Uveitis, Skleritis, } \\
\text { Optikusneuritis und } \\
\text { okuläre Myositis }\end{array}$ & $\begin{array}{l}\text { Uveitis, Optikusneuritis } \\
\text { und Endophthalmitis }\end{array}$ & $\begin{array}{l}\text { Uveitis, Optikusneuritis, } \\
\text { Endophthalmitis, } \\
\text { Korneainfiltrate, retinale } \\
\text { Toxizität und } \\
\text { Ophthalmoplegie }\end{array}$ & Optikusneuritis & Optikusneuritis \\
\hline
\end{tabular}

depunkt und trugen dazu bei, dass Adalimumab von der US-amerikanischen Food and Drug Administration (FDA) und der Europäischen Arzneimittel-Agentur (EMA) zur Behandlung der nicht infektiösen intermediären, posterioren und Panuveitis bei Erwachsenen und Kindern über 2 Jahren zugelassen wurde. Darüber hinaus waren sie ausschlaggebend für die Zulassung durch das National Institute for Health and Care Excellence (NICE) im Vereinigten Königreich.

Eine kürzlich veröffentlichte systematische Übersichtsarbeit ergab, dass Adalimumab das Risiko eines Therapieversagens um 43 bis $75 \%$ verringerte und eine erfolgversprechende therapeutische Option darstellen könnte, um bei nicht infektiöser Uveitis die Entzündungsaktivität zu senken, die Sehschärfe zu verbessern und Kortikosteroide einzusparen [20].

Zwar gilt der Einsatz der anderen Anti-TNF-Therapien bei Uveitis als Off-Label-Anwendung, doch werden sie weiterhin zur Kontrolle der bei systemischen Erkrankungen auftretenden Augenentzündung [21,22] eingesetzt und sie sind besonders bei Morbus Behçet $[23,24]$ und juveniler idiopathischer Arthritis wirksam [25]. Die Erfahrungen mit Golimumab [26] und Certolizumab [27] zur Behandlung von Augenentzündungen sind begrenzt, doch ermutigend.

\section{Okuläre Nebenwirkungen}

Biologische TNF- $\alpha$-Antagonisten sind zu den wichtigsten Wirkstoffen in der Behandlung von Patienten mit chronischen immunvermittelten Krankheiten geworden und kommen seit 10 Jahren zunehmend zum Einsatz. Dies hat zur Folge, dass verschiedene Nebenwirkungen, darunter auch okuläre Ereignisse, im Zusammenhang mit ihrer Anwendung berichtet wurden [4]. Es liegen Berichte vor, nach denen TNF-Inhibitoren paradoxerweise entgegen ihrer therapeutischen Wirkung mit dem Auftre- ten oder Wiederauftreten von entzündlichen Augenerkrankung assoziiert sind, insbesondere unter Etanercept [28]. Meist handelt es sich dabei um eine Uveitis anterior (UA), doch wurde auch die Auslösung einer intermediären Uveitis [29], einer Uveitis posterior [30], einer Skleritis [31] und sogar einer orbitalen Myositis [32] berichtet. Andere okuläre Nebenwirkungen sind Blepharitis und Ektropium [33], Optikusneuritis [28], Verschlechterung des Sehvermögens [34], Diplopie [34-36], Endophthalmitis [37, 38] und «sarcoid-like granulomatosis» [39], Panuveitis [40] oder retinale Vaskulitis [41].

Die Bedeutung von Anti-TNF-Wirkstoffen für die Pathogenese okulärer Nebenwirkungen bei der Anwendung zur Behandlung von Krankheiten, bei denen eine Augenbeteiligung Teil des klinischen Manifestationsspektrums ist, ist umstritten. Eine nach Behandlungsbeginn neu auftretende Uveitis und die Entwicklung einer Uveitis bei fehlender Gelenkaktivität [28, 32, 42, 43] sprechen dafür, dass bei der okulären und artikulären Entzündung unterschiedliche ätiopathogenetische Mechanismen eine Rolle spielen. Demgegenüber scheint die Rolle von Anti-TNF-Wirkstoffen bei Erkrankungen, bei denen eine Augenbeteiligung nicht als Teil des klinischen Manifestationsspektrums angesehen wird, wie etwa bei rheumatoider Arthritis (RA), klarer zu sein.

Es wurde die Hypothese aufgestellt [44], dass es bei einzelnen RAPatienten unter Anti-TNF-a zu einer Uveitis kommen kann, und dass die Dosierungen in der Lage sind, die Gelenkmanifestationen zu kontrollieren, jedoch nicht ausreichen, um die Uveitis zu kontrollieren [17], oder dass bei einigen der mit Anti-TNF-Wirkstoffen behandelten Patienten eine nicht erkannte infektiöse Ursache eine Erklärung für die Uveitis sein könnte [45]. Die Bedeutung von Anti-TNF-Wirkstoffen bei der Induktion einer entzündlichen Augenerkrankung (inflammatory ocular disease, IOD) wurde auch von Wendling et al. [29] in einer Übersichtsarbeit von 152 Fällen angezweifelt. Diese berichtete, dass es bei den 
meisten Patienten zu einer Abheilung der Uveitis kam - auch bei denjenigen, bei denen die Anti-TNF-Therapie fortgesetzt wurde. Dies spricht für einen schwachen Einfluss dieser Medikamente. Es liegen jedoch zunehmend Hinweise vor, nach denen diese Therapien mit einer paradoxen Nebenwirkung mit Augenentzündung assoziiert sind, was stark auf eine echte Ursache-Wirkungs-Beziehung hindeutet. Zudem klang die Uveitis in vielen Fällen ab, nachdem die Behandlung abgesetzt wurde [46], und eine erneute Exposition führte zu einem Wiederauftreten der Erkrankung [28, 47-49].

\section{Mechanismen der Immunogenität}

Die Mechanismen, über die TNF- $\alpha$-Inhibitoren paradoxe Entzündungseffekte auslösen, sind unklar. Eine Hypothese bezieht sich auf die inverse und ineinandergreifende Beziehung der TNF- $\alpha$ und Interferon-Spiegel (sowohl Interferon $\alpha$ als auch $\gamma$ ) und die Bedeutung erhöhter Interferonspiegel bei der Entstehung von Autoimmunerkrankungen [50]. Anderen Vermutungen zufolge könnte die Hemmung von TNF- $\alpha$ direkt oder indirekt zu einer Erhöhung der Infektionsrate durch Organismen führen, die an der Entstehung nicht verkäsender Granulome beteiligt sind [39]. Und schließlich könnte eine Dosisabhängigkeit bestehen, da sich einige paradoxe okuläre Nebenwirkungen möglicherweise mit einem Hochdosisschema im Vergleich zur Standarddosierung, wie sie bei entzündlichen rheumatischen Erkrankungen gegeben wird, kontrollieren lassen [5].

Die Datenbank von Spontanberichten über Uveitis unter AntiTNF-Therapie in den USA [28] zeigte, dass der lösliche TNF- $\alpha$ Rezeptor (Etanercept) gegenüber den monoklonalen Antikörpern mit den meisten Fällen assoziiert war (43 Fälle unter Etanercept, 14 unter Infliximab und 2 unter Adalimumab). Nach Normalisierung für die geschätzte Anzahl der mit dem jeweiligen Medikament behandelten Patienten war Etanercept weiterhin mit einer höheren Zahl an Uveitis-Fälle verbunden $(p<0,01)$, und dies auch nachdem Patienten, deren Grunderkrankung mit einer Uveitis assoziiert war, ausgeschlossen worden waren. In einer französischen Studie [29], in der 153 Fälle erfasst wurden, traten 84\% der Fälle im Zusammenhang mit Etanercept auf. Dies spricht dafür, dass bei diesen beiden Medikamentenklassen unterschiedliche immunologische Eigenschaften beteiligt sind.

Verschiedene Hypothesen versuchen, diesen Unterschied zu erklären. In Hinblick auf die Pharmakokinetik [51] weist Etanercept eine kürzere Halbwertszeit und ein kleineres Verteilungsvolumen sowie eine schnellere Clearance auf als Adalimumab und Infliximab. Es liegen Hinweise vor, nach denen nur Infliximab die Apoptose aktivierter Makrophagen und T-Zellen induzieren kann und daher, theoretisch, einen größeren Einfluss auf die Steuerung der Immunreaktion hat [52]. Zudem sind Infliximab und Adalimumab in der Lage, sowohl TNFRp55- als auch TNFRp75-vermittelte Ereignisse zu blockieren, wohingegen Etanercept nur auf TNFRp55 wirkt [53]. Ferner wurden Unterschiede beim reverse Signalling und bei der Zytokinmodulation festgestellt [54]. Einige Autoren vermuteten, dass Etanercept einen heraufregulierenden Effekt auf die T-Zell-Zytokin-Reaktionen hat, während Infliximab herunterregulierend wirkt [55-57]. Und schließlich könnte es sein, dass Etanercept als löslicher Rezeptor die Halbwertzeit von intraokulärem TNF-a verlängert, wodurch die Uveitis stimuliert wird bis der Rezeptor-Ligand-Komplex aus dem Auge entfernt wird [57].

\section{Ergebnisse}

\section{Etanercept}

Etanercept $\left(\right.$ Enbrel $^{\circledR}$, Amgen Wyeth, Immunex Corporation, Thousand Oaks, CA, USA) war der erste Anti-TNF- $\alpha$-Wirkstoff, der 1998 von der FDA zugelassen wurde. Es handelt sich dabei um ein humanisiertes, rekombinantes dimeres Fusionsprotein von einem menschlichen Fc-Molekül und zwei p75-TNF-Rezeptoren. Etanercept verhindert die Bindung von TNF- $\alpha$ und TNF- $\beta$ an TNF-Rezeptoren auf der Zelloberfläche, indem es an diese zirkulierenden Faktoren bindet. Anders als andere Wirkstoffe seiner Klasse bewirkt Etanercept keine Lyse der TNF-bildenden Zellen.

\section{Uveitis}

In einer registerbasierten US-amerikanischen Studie fanden sich 43 Fälle von Uveitis im Zusammenhang mit Etanercept [28]. Andere Studien zeigten diesen Zusammenhang ebenfalls [30, 49]. Im Vergleich zu den anderen Anti-TNF- $\alpha$-Medikamenten bestand bei Etanercept eine höhere Assoziation mit Uveitis.

Mögliche Gründe für dieses Phänomen wurden bereits weiter oben in dieser Übersichtsarbeit erörtert. Eine Erklärung könnte sein, dass die Hemmung mit Infliximab sowohl TNFRp55- als auch TNFRp75-vermittelte Ereignisse blockiert, wohingegen Etanercept den TNFRp75-vermittelten Signalweg zumindest teilweise intakt lässt [53]. In einer anderen Veröffentlichung [58] wurde festgestellt, dass Infliximab einen stabilen Komplex mit sTNF bildet, während dies bei Etanercept nicht der Fall ist. Zudem könnte die Fähigkeit des löslichen p75-Rezeptors (Etanercept), TNF effektiv zu binden und anschließend freizusetzen, dazu beitragen, die zirkulierende Halbwertszeit von TNF zu verlängern [59].

\section{Skleritis}

Die Skleritis ist eng mit RA assoziiert, und Berichten zufolge liegt die Gesamtprävalenz der Skleritis bei RA-Patienten zwischen 0,67 und $6,3 \%[60,61]$.

Le Garrec at al [62]. berichteten über 3 Patientinnen, die eine erstmalige Episode einer unilateralen Skleritis oder eine akute Uveitis anterior entwickelten, während sie zur Behandlung einer rheumatologischen Erkrankung Etanercept erhielten. Die Entzündung ging erst zurück, als Etanercept abgesetzt wurde.

Eine weitere spätere Studie [31] beschrieb 3 Fälle von Skleritis im Zusammenhang mit der Anwendung von Etanercept bei RA. Die okuläre Entzündung besserte sich nach Absetzen der Behandlung; weitere Rezidive traten nicht auf. In einem Fall führte die erneute Exposition zum Wiederauftreten der okulären Symptome. Bei der Überprüfung der Literatur fanden die Autoren weitere 8 Fälle von Skleritis im Zusammenhang mit der Anwendung von Etanercept. 


\section{Optikusneuritis}

Verschiedene demyelinisierende Erkrankungen wurden in Verbindung mit Anti-TNF- $\alpha$-Wirkstoffen berichtet. Zahlreiche Arbeiten wiesen den Zusammenhang zwischen einer Optikusneuritis und Etanercept nach [28, 63-66]. Patienten, die mit Anti-TNF- $\alpha$ Wirkstoffen behandelt werden, sollten engmaschig auf ophthalmologische oder neurologische Symptome überwacht werden.

\section{Okuläre Myositis}

Bei der okulären Myositis handelt es sich um eine seltene Erkrankung, die durch eine Entzündung einzelner oder mehrerer extraokulärer Augenmuskeln mit schmerzhafter Diplopie und/oder Ophthalmoplegie gekennzeichnet ist. In der Literatur finden sich 2 Berichte über eine okuläre Myositis unter Etanercept zur Behandlung einer RA $[67,68]$.

\section{Infliximab}

Infliximab (Remicade ${ }^{\circledR}$, Janssen Biotech, Inc., Horsham, PA, USA) ist ein chimärer murin/humaner monoklonaler Antikörper, der sowohl zirkulierenden als auch membrangebundenen TNF- $\alpha$ bindet und neutralisiert und der 1999 als zweiter AntiTNF- $\alpha$-Wirkstoff von der FDA zugelassen wurde.

\section{Uveitis}

In derselben, bereits für Etanercept erwähnten, registerbasierten Studie wurden 12 Fälle von Uveitis im Zusammenhang mit Infliximab festgestellt [28]. Eine retrospektive Studie fand 5 Fälle von neu aufgetretener Uveitis bei Patienten, die mit Infliximab behandelt wurden [29]. In einem Fallbericht wurde der Fall eines Patienten mit ulzerativer Kolitis beschrieben, der wahrscheinlich eine dosisabhängige Uveitis unter der Behandlung mit Infliximab entwickelte [69].

\section{Optikusneuritis}

Die genaue Anzahl der Fälle von Optikusneuritis infolge einer Therapie mit Infliximab oder anderen TNF-Hemmern ist nicht bekannt, scheint aber sehr viel höher zu sein als in der Literatur beschrieben [70]. Es liegen zahlreiche Berichte über das Auftreten einer Optikusneuropathie und -neuritis unter der Behandlung mit Infliximab vor [34, 71-79]. Die meisten Fälle manifestieren sich mit bilateraler und retrobulbärer Beteiligung, doch wurden unterschiedliche Muster beschrieben.

\section{Endophthalmitis}

Immunschwächeerkrankungen und immunsuppressive Therapien sind bekannte Risikofaktoren für die Entwicklung einer Endophthalmitis [80]. Verschiedene Fallberichte berichteten über eine Endophthalmitis unter der Behandlung mit Infliximab [38, 81-83]. Jin et al. vermuteten, dass die Therapie mit Anti-TNF- $\alpha$-Wirkstoffen bei der Migration von Zilien in den Bulbus und der Entwicklung einer Endophthalmitis eine Rolle spielen könnte. Ein weiterer möglicher Mechanismus, der zu einem erhöhten Endophthalmitis-Risiko unter der Behandlung mit Anti-TNF- $\alpha$ beiträgt und beurteilt werden sollte, ist die Skleritis-bedingte Verdünnung der Sklera [81].

\section{Adalimumab}

Adalimumab (Humira ${ }^{\circledR}$, AbbVie Inc., North Chicago, IL, USA) ist ein vollständig humanisierter monoklonaler Antikörper gegen TNF- $\alpha$. Seit seiner Zulassung durch die FDA 2002 hat er sich zu einem der meist verordneten Biologika in den USA entwickelt [84, 85]. Er ist für 12 Indikationen zugelassen, einschließlich nicht infektiöse intermediäre, posteriore und Panuveitis. Bis jetzt erfolgt die Behandlung der Uveitis mit Adalimumab immer noch systemisch; es wurden jedoch Untersuchungen in Hinblick auf eine lokale Therapie mit intravitrealen Injektionen durchgeführt [8690]. Obwohl in den meisten Studien die Sicherheit nachgewiesen wurde, ist dieser Aspekt weiterhin strittig, und es sind weitere Forschungsarbeiten erforderlich, um dieses Verfahren in Zukunft praktikabel zu machen.

\section{Uveitis}

Zwar traten in der registerbasierten Studie von Lim et al. [28] unter der Adalimumab-Therapie weniger Uveitis-Fälle auf (2 Fälle), doch bestand bei den anderen Anti-TNF- $\alpha$-Wirkstoffen kein signifikanter Unterschied zwischen Adalimumab und Infliximab $(p$ $>0,5, \mathrm{OR}=1,6)$. Seve et al. [41] berichteten über den Fall einer Patientin mit RA, die während einer 3-jährigen Behandlung mit Adalimumab eine bilaterale sarkoidassoziierte Panuveitis mit granulomatösen keratischen Präzipitaten, Periphlebitis und eine periphere multifokale Choroiditis entwickelte.

\section{Hornhautfiltrate}

Wir fanden einen Fallbericht [91] einer Patientin mit Morbus Crohn, bei der es $36 \mathrm{~h}$ nach einer Adalimumab-Injektion zu bilateralen peripheren Hornhautinfiltraten mit den charakteristischen Zeichen von Immuninfiltraten gekommen war. Die Symptome und Infiltrate gingen nach Gabe von topischen Kortikosteroiden zurück, traten jedoch in den folgenden Wochen nach jeder Adalimumab-Injektion erneut auf.

\section{Optikusneuritis/-neuropathie}

Auch Lim et al. [28] fanden in ihrer Studie weniger Fälle von Optikusneuritis unter Adalimumab (4 Fälle gegenüber 50 Fällen unter Etanercept und 40 Fällen unter Infliximab). Andere Autoren berichteten ebenfalls über Fälle von Optikusneuritis im Zusammenhang mit der Adalimumab-Therapie [92-96].

\section{Retinale Toxizität}

Eine Studie zur Beurteilung der okulären Toxizität steigender Dosen von intravitreal verabreichtem Adalimumab (Humira) am Kaninchenauge [87] ergab, dass die Verabreichung höherer Dosen mit einer Entzündungsreaktion und Netzhautnekrose assoziiert war. In unserer Arbeit fanden wir einen Bericht über Netzhautnekrose nach einer Adalimumab-Therapie beim Menschen [97]. Unter Umständen war dieser Fall tatsächlich durch eine virale Retinitis (möglicherweise Varicella-Zoster-Virus) bedingt. Eine andere Studie [98] berichtete über eine Patientin, die wegen Morbus Crohn mit Adalimumab behandelt wurde, und bei der einen Monat nach Behandlungsbeginn eine diffuse bilaterale Retinopa- 
thie auftrat, die nur durch elektrophysiologische Untersuchungen festgestellt wurde.

\section{Endophthalmitis}

Es fand sich nur ein Fallbericht über Endophthalmitis im Zusammenhang mit einer Adalimumab-Therapie [37]. Dieser Bericht steht im Einklang mit der Literatur zu P. acnes, das bei immungeschwächten Patienten pathologische Reaktionen und eine Endophthalmitis verursachen kann [99].

\section{Ophthalmoplegie}

In der Literatur findet sich ein Fallbericht über eine internukleäre Ophthalmoplegie bei einer Frau mit anamnestisch bekanntem Morbus Crohn, die ein Taubheitsgefühl im Gesicht, verschwommenes Sehen und Diplopie im rechten Auge entwickelte [100].

\section{Golimumab und Certolizumab}

Golimumab (Simponi ${ }^{\circledR}$ und Simponi Aria ${ }^{\circledR}$, Janssen Biotech, Inc., Horsham, PA, USA) ist ein vollständig humaner monoklonaler Antikörper, der 2009 von der FDA zugelassen wurde. Certolizumab pegol (Cimzia ${ }^{\circledR}$, UCB, Inc., Smyrna, GA, USA) ist zur Behandlung von mittelschwerer bis schwerer RA, PsoriasisArthritis und Morbus Crohn zugelassen. Anders als bei den anderen monoklonalen Anti-TNF-Antikörpern handelt es sich bei diesem Wirkstoff um ein polyethylenglykolisiertes Fab-Fragment.

$\mathrm{Da}$ es sich hierbei um neuere Arten von Anti-TNF- $\alpha$-Wirkstoffen handelt, liegen für beide noch sehr wenige Hinweise auf okuläre Nebenwirkungen vor. Bei der Überprüfung des National Registry of Drug Induced Ocular Side Effects im Rahmen der SABER-Studie [101] fanden die Autoren 5 Fälle von Optikusneuritis im Zusammenhang mit Golimumab oder Certolizumab. In derselben Studie traten die meisten Fälle unter Etanercept $(n=169)$ auf, wohingegen unter Infliximab und Adalimumab weniger Fälle berichtet wurden ( $n=122$ bzw. $n=55)$.

\section{Biosimilars}

Das Spektrum an TNF-Blockern wächst stetig. Seit dem Auslaufen des Patentschutzes für Infliximab 2015 erhielten zahlreiche Biosimilars die Zulassung der EMA und FDA und werden in der klinischen Praxis angewendet. Seit 2017 sind auch Biosimilars für Adalimumab erhältlich.

Die Erfahrungen aus der klinischen Praxis und Daten zu diesen neuartigen Arzneimitteln sind immer noch begrenzt; eine systematische Übersichtsarbeit zeigte jedoch, dass Biosimilars ein ähnliches Sicherheits- und Wirksamkeitsspektrum aufweisen wie die Originalmedikamente [102]. Daher sollte bei der Therapie mit Biosimilars eine engmaschige Kontrolle auf die mit dem originalen Anti-TNF-Wirkstoff assoziierten okulären Nebenwirkungen erfolgen. Eine kürzlich durchgeführte ophthalmologische Studie zeigte, dass bei der Therapieumstellung vom originalen Anti-TNF- $\alpha$-Wirkstoff auf das entsprechende Biosimilar die klinische Wirksamkeit bei Patienten mit nicht infektiöser Uveitis erhalten bleibt [103].

\section{Diskussion}

Anti-TNF-Biopharmazeutika haben sich als wichtige Entwicklung in der Behandlung immunvermittelter Krankheiten erwiesen, doch sind bei Entscheidung, bei einem Patienten eine AntiTNF-Therapie einzuleiten, potenziell schwerwiegende Nebenwirkungen zu berücksichtigen.

Anti-TNF- $\alpha$-Wirkstoffe kommen zunehmend in der Behandlung von Augenentzündungen zum Einsatz, und die meisten Daten, die ihre Anwendung bei nicht infektiöser Uveitis stützen, liegen für Adalimumab und Infliximab vor. Diese Wirkstoffe gelten als geeignete Therapie für die meisten Formen von nicht infektiöser Uveitis, während sie bei Uveitis im Zusammenhang mit Morbus Behçet und juveniler idiopathischer Arthritis als First-Line-Therapie angesehen werden können [104]. Durch schwerwiegende potenzielle Nebenwirkungen, wie das Risiko opportunistischer Infektionen, die Reaktivierung einer latenten Tuberkulose und die Entwicklung eines Malignoms kann ihre Anwendung in der Ophthalmologie allerdings eingeschränkt sein.

Darüber hinaus wurde über ein breites Spektrum an paradoxen Nebenwirkungen berichtet [5]. Etliche Studien deuten darauf hin, dass Anti-TNF- $\alpha$-Wirkstoffe und TNF- $\alpha$ bei der Auslösung oder Verschlimmerung eines zugrunde liegenden Entzündungsprozesses eine Rolle spielen könnten [105-108]. Von den verschiedenen Theorien, die vorgeschlagen wurden, um paradoxe Nebenwirkungen zu erklären, ist die Hypothese eines gestörten Zytokingleichgewichts vermutlich am realistischsten [5]. Bei chronischen immunvermittelten Krankheiten sind zahlreiche immunologische Mechanismen beteiligt. Die zusätzliche Gabe von biologischen Wirkstoffen verändert das Zytokinmilieu und fördert so neue Signalwege, wodurch ein günstiges immunologisches Umfeld für paradoxe Nebenwirkungen entsteht.

Vor diesem Hintergrund können viele der in dieser Übersichtsarbeit berichteten okulären Nebenwirkungen als paradoxe Wirkung der Anti-TNF-Therapie angesehen werden, insbesondere die Uveitis. In der vorliegenden Arbeit fanden wir umfangreiche Daten, nach denen ein Zusammenhang zwischen neu aufgetretener Uveitis und Anti-TNF-Therapie bei rheumatischen Erkrankungen besteht, insbesondere bei Patienten mit Spondyloarthritis und unter Etanercept [28, 29]. Bei Auftreten einer Uveitis in Verbindung mit einer Anti-TNF- $\alpha$-Therapie ist es wichtig, auch andere Ursachen im Zusammenhang mit der Anwendung dieser Wirkstoffe in Betracht zu ziehen, da diese Art der Therapie mit einem erhöhten Infektionsrisiko, insbesondere granulomatösen Infektionen, assoziiert ist [109]. Zudem können entzündliche Augenerkrankungen als Zeichen der systemischen Grunderkrankung auftreten. Daher standen in dieser Übersichtsarbeit Fälle mit dosisabhängiger Reaktion, zeitlichem Zusammenhang, positiver Re-Exposition oder neu aufgetretener Erkrankung im Vordergrund.

Das Bewusstsein für mögliche Nebenwirkungen der Behandlung sollte erhöht werden, doch bleibt abzuwarten, ob qualitativ hochwertigere Daten den vermuteten Zusammenhang zwischen TNFHemmern und dem Auftreten einer Uveitis untermauern. Wir erfassten hauptsächlich Fallberichte und retrospektive Studien, 
was aufgrund der Vielzahl an Uveitis-Ursachen und ihres möglichen Zusammenhangs mit den autoimmunen Grunderkrankungen, die mit Anti-TNF- $\alpha$-Wirkstoffen behandelt werden, Verzerrungsfehler zur Folge haben kann. Zukünftige groß angelegte, prospektive Studien nach der Markteinführung, die auch mit herkömmlichen Therapien behandelte Patienten als Kontrollen einschließen, können helfen, das tatsächliche Uveitis-Risiko von Patienten, die biologische Therapien erhalten, zu beurteilen.

Eine weitere okuläre Nebenwirkung, die durchgängig mit der Anti-TNF- $\alpha$-Therapie assoziiert war, ist die Optikusneuritis. Der Zusammenhang zwischen einer Anti-TNF- $\alpha$-Therapie und dem Auftreten demyelinisierender Erkrankungen ist weiter unklar, doch lässt die zeitliche Abfolge der klinischen Ereignisse in einer Reihe von Fällen einen solchen vermuten. In einer randomisierten, placebokontrollierten Studie mit 168 Patienten mit Multipler Sklerose (MS) (unter Ausschluss von Patienten mit schnell fortschreitender MS), die Lenercept erhielten, waren eine Zunahme der Schubhäufigkeit und ein Trend zu schwereren Schüben im Vergleich zu Placebo zu beobachten [110].

Die Optikusneuritis ist eine schwerwiegende Komplikation, die zu einem irreversiblen Sehverlust führen kann. Deshalb sollten Patienten, die einen TNF- $\alpha$-Antagonisten erhalten, engmaschig auf die Entwicklung ophthalmologischer oder neurologischer Symptome überwacht werden. Klinisches Bild, serologische und röntgenologische Untersuchungsergebnisse sowie ein schnelles Ansprechen auf das Absetzen potenziell schädlicher Medikamente sprechen für eine Anti-TNF-Antagonisten-assoziierte Optikusneuritis [63, 65, 71].

Wird die Diagnose Optikusneuritis gestellt, sollte eine Behandlung mittels Kortikosteroid-Stoßtherapie gefolgt von oralen Kortikosteroiden eingeleitet werden. Die Überprüfung der Literatur ergab, dass es bei 9 von 15 Patienten nach der Behandlung zu einer vollständigen Rückbildung kam [65]. Darüber hinaus sollte eine sorgfältige Abwägung erfolgen, um Anti-TNF-Therapien bei Patienten mit einer anamnestisch bekannten demyelinisierenden Erkrankung zu vermeiden.

In einer nationalen französischen Untersuchung [34] stellten die Autoren fest, dass die Krankheit trotz Absetzen der Behandlung persistieren kann. Dies spricht dafür, dass Anti-TNF- $\alpha$-Wirkstoffe einen Demyelinisierungsprozess auslösen können, der sich anschließend unabhängig weiter entwickelt. Eine große US-amerikanische multizentrische Kohortenstudie zum gleichen Thema (SABER) [101] untersuchte die Inzidenz der Optikusneuritis bei Patienten, bei denen eine biologische Therapie mit Anti-TNFWirkstoffen begonnen wurde. Die von den Autoren durchgeführten Sensitivitätsanalysen deuten darauf hin, dass die Häufigkeit der Optikusneuritis bei Patienten, die mit Anti-TNF-Therapien behandelt wurden, ähnlich hoch ausfiel wie bei vergleichbar stark betroffenen Patienten, die nicht biologische immunsuppressive Therapien erhielten.

Gemäß den Behandlungsstrategien für okuläre Nebenwirkungen ist das auslösende Medikament abzusetzen. Eine geeignete Option ist die Substitution durch Kortikosteroide [65-67, 71], die in vielen Fällen unter Umständen allerdings nur eine kurzfristige
Lösung darstellt. Eine weitere Möglichkeit, die in der Literatur als wirksam angesehen wird, ist die Umstellung auf andere immunsuppressive Medikamente [63, 96, 111] oder andere Anti-TNFWirkstoffklassen [30], wobei es nach Einführung eines anderen Anti-TNF-Wirkstoffs jedoch zu einem Rezidiv kommen kann [34]. Berichten zufolge ist die Vorbehandlung mit Kortikosteroiden vor der Anti-TNF-Infusion mit positiven Ergebnissen verbunden, wenn ein Absetzen der Therapie nicht möglich ist [69].

\section{Schlussfolgerung}

Zwar liegen immer mehr Daten zu okulären Nebenwirkungen im Zusammenhang mit der Anti-TNF-a-Therapie vor, doch stammen die meisten verfügbaren Daten aus nicht kontrollierten Studien, was fundierte Schlussfolgerungen unmöglich macht. Trotz der eingeschränkten Qualität der verfügbaren Daten ist die vorliegende Übersichtsarbeit wichtig, da sie die aktuelle Datenlage zusammenträgt und mögliche Nebenwirkungen und paradoxe Effekte der Anti-TNF-Wirkstoffe beleuchtet, die Rheumatologen und Augenärzte im Blick haben sollten.

Bei der Diagnose und Behandlung müssen Rheumatologen und Augenärzte zusammenarbeiten. In manchen Fällen macht eine persistierende und/oder rezidivierende Augenerkrankung ein Absetzen der Therapie erforderlich.

Unsere Ergebnisse verändern letztlich nicht die derzeitigen Indikationen für die Anwendung von Biologika. Vielmehr empfehlen sie eine engmaschige Kontrolle des Augenstatus bei Patienten, die Anti-TNF-Wirkstoffe erhalten, und stoßen die Diskussion über die Notwendigkeit einer höheren Evidenzqualität zu diesem Thema an.

\section{Autorenbeiträge}

F. S. überprüfte die Literatur und erstellte das Manuskript. C. P. entwickelte das Konzept für die Arbeit und nahm die kritische Prüfung des Manuskripts vor. Alle Autoren haben die finale Fassung des Manuskripts gelesen und freigegeben.

\section{Finanzierung der Studie}

Die vorliegende Arbeit wurde vom National Institute for Health Research (NIHR) Biomedical Research Centre at Moorfields Eye Hospital NHS Foundation Trust und vom UCL Institute of Ophthalmology finanziert.

\section{Disclosure Statement}

C. P. erhielt Vortragshonorare von AbbVie und arbeitet als Berater für Alimera Sciences.

\section{Lizenzangabe}

Susanna FN, Pavesio C: A review of ocular adverse events of biological anti-TNF drugs. J Ophthalmic Inflamm Infect. 2020;10:11. (DOI: 10.1186/ s12348-020-00202-6). ${ }^{\circledR} 2020$ The Author(s). (Übersetzung; Abkürzungen, Danksagung, Verfügbarkeit der Daten und Materialien, Genehmigung durch die Ethikkomission und Einwilligung in die Teilnahme, Zustimmung zur Veröffentlichung und Publisher's Note gekürzt), lizensiert unter CC BY 4.0 (https://creativecommons.org/licenses/by/4.0/deed.de).

\section{Literatur}

Die Literatur ist unter www.karger.com/doi/10.1159/000509813 abrufbar. 